\title{
The distal form of spinal muscular atrophy: an unusual case demonstrating the $\frac{\frac{\bar{c}}{\bar{\omega}}}{\frac{\bar{v}}{2}}$ intermediate variety
}

\author{
D. A. ISENBERG* \\ M.B., B.S., M.R.C.P. \\ PAULINE A. KAHN† \\ M.B., Ch.B. \\ * Department of Rheumatology, University College Hospital, London WC1 and †Department of Neuropathology \\ Whittington Hospital, London $N 19$
}

\section{Summary}

A 14-year-old boy with a long history of distal muscle weakness affecting primarily and predominantly the upper limbs is described. There is a family history of pes cavus and congenital dislocation of the hip. Electromyography and histopathological studies of skeletal muscle showed conclusive evidence of a neurogenic muscular disorder, and excluded primary muscle disease. The muscle biopsy showed group atrophy. As many target fibres which are identical to structured cores were a prominent feature of the biopsy, central core disease was considered. However, it was concluded on clinical, neurophysiological and histological evidence that the patient was suffering from distal spinal muscular atrophy of an intermediate type designated by previous authors.

A review of the current concepts of distal muscle weakness is included.

\section{Introduction}

It has become apparent within the past few years that patients presenting with predominantly distal muscle weakness may be suffering from a variety of clinical syndromes. The earliest descriptions of patients presenting with peroneal muscular atrophy were those of Charcot and Marie (1886) and Tooth (1886). They described a disease presenting in childhood causing progressive weakness and atrophy of distal muscles and some sensory deficit, affecting the lower limbs and much later, the upper limbs. Subsequent clinical descriptions indicated that a wide variety of variants existed. A more ordered classification became possible with the introduction of nerve conduction studies in the mid-nineteenfifties.

In 1968 Dyck and Lambert defined two broad groups. Group I comprised cases with severely

\footnotetext{
Correspondence and requests for reprints to Dr Pauline Kahn.
}

reduced motor and sensory nerve conduction velocity, sometimes with hypertrophic nerves. Some cases of of Charcot-Marie-Tooth disease fall into this group. $₫$ In Group II nerve conduction velocity was relatively 윽 preserved and this group included the 'neuronal' form of Charcot-Marie-Tooth disease. Subsequently, $\stackrel{\mathcal{D}}{\mathbb{2}}$ these two groups have been termed hereditary motor $O$ and sensory neuropathy (HMSN) types I and II (Thomas and Calne, 1974; Thomas, Calne and Stewart, 1974; Dyck, 1975).

Harding and Thomas (1980) reviewed 262 cases of $\frac{0}{\oplus}$ peroneal muscular atrophy. A total of 228 cases were ? classified as HMSN types I or II. The remaining 3 \% cases had the clinical syndrome of peroneal muscular atrophy without sensory loss, which displayed electromyographic evidence of denervation in the distal musculature, normal or slightly reduced motor nerve conduction velocities and normal sensory action potentials. These patients were classified as examples of distal spinal muscular atrophy.

The spinal muscular atrophies are a heterogeneous group of disorders which may present from birth to adult life, in which progressive degeneration of lower motor neurones occurs leading to neuropathic atrophy of muscles. It is usually the proximal muscles which are affected but occasionally distal muscles of the lower limbs are involved (Martin-Sneesens, 1962; Dyck and Lambert, 1968; Brust, Lovelace and Devi, 1978). Very rarely the distal muscles of the upper limbs are predominately affected (O'Sullivan and McLeod, 1978). We describe a case which supports the existence of an 'intermediate' distal spinal muscular atrophy (SMA) in which the distal muscles of both arms and legs are involved from the earliest stages of the disease.

\section{Case report}

A 14-year-old schoolboy presented with a history of weakness in his hands for as long as he could 


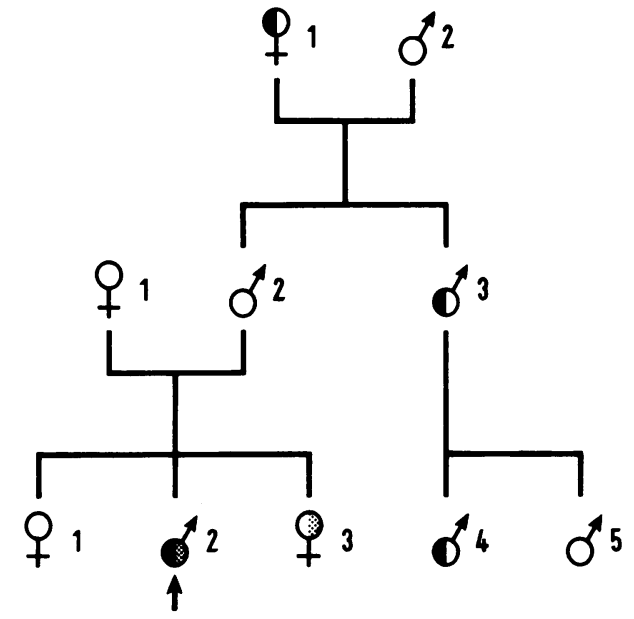

$\uparrow$ Propositus 0 Pes cavus Congenital hip dislocation

FIG. 1. Family tree showing the presence of pes cavus and congenital hip dislocation.

remember. For four years he had been particularly aware of the problem as it had prevented him from normal participation in gymnastics. For two years he had been unable to lift heavy objects and found writing increasingly difficult. He had found difficulty in doing up his shirt buttons for one month. He also complained of intermittent cramps in the hands and forearms for one year. He had not noted any exacerbation of his symptoms during cold weather. He did not walk until age 17 months and had 2 operations when aged 3 and 6 years, for a left congenital hip dislocation. He had been aware of slight weakness of feet and ankles for about 4 years, though he was not greatly restricted by this. There was a family history of pes cavus and congenital hip dislocation (Fig. 1). Examination of the patient's parents and siblings revealed no abnormalities apart from the scar of an operation for congenital hip dislocation in the younger sister (case III 3 ).

On examination, pes cavus, a slight kyphoscoliosis and the scar of his operations were evident. There were no abnormalities in his cardiovascular, respiratory or abdominal systems. His cranial nerves were normal. In his upper limbs slight wasting of the deltoids and forearm muscles and more marked wasting of the small muscles of the hands (Fig. 2) was evident. Tone was a little reduced bilaterally and there was no evidence of myotonia. Power was reduced to 4+ (MRC grading) in the proximal muscles and 4- in the flexors and extensors of the wrist and in the small muscles of the hand. Deep tendon reflexes were uniformly absent. The abdominal reflexes were present. There were no sensory abnormalities. In the lower limbs mild calf hypertrophy was present. There was no muscle wasting or alteration in tone. Power was $4+$ in all muscle groups except in the ankle dorsiflexors and evertors of the feet which were grade 4. Deep tendon reflexes were absent and the plantar responses were both flexor. Sensation was normal.

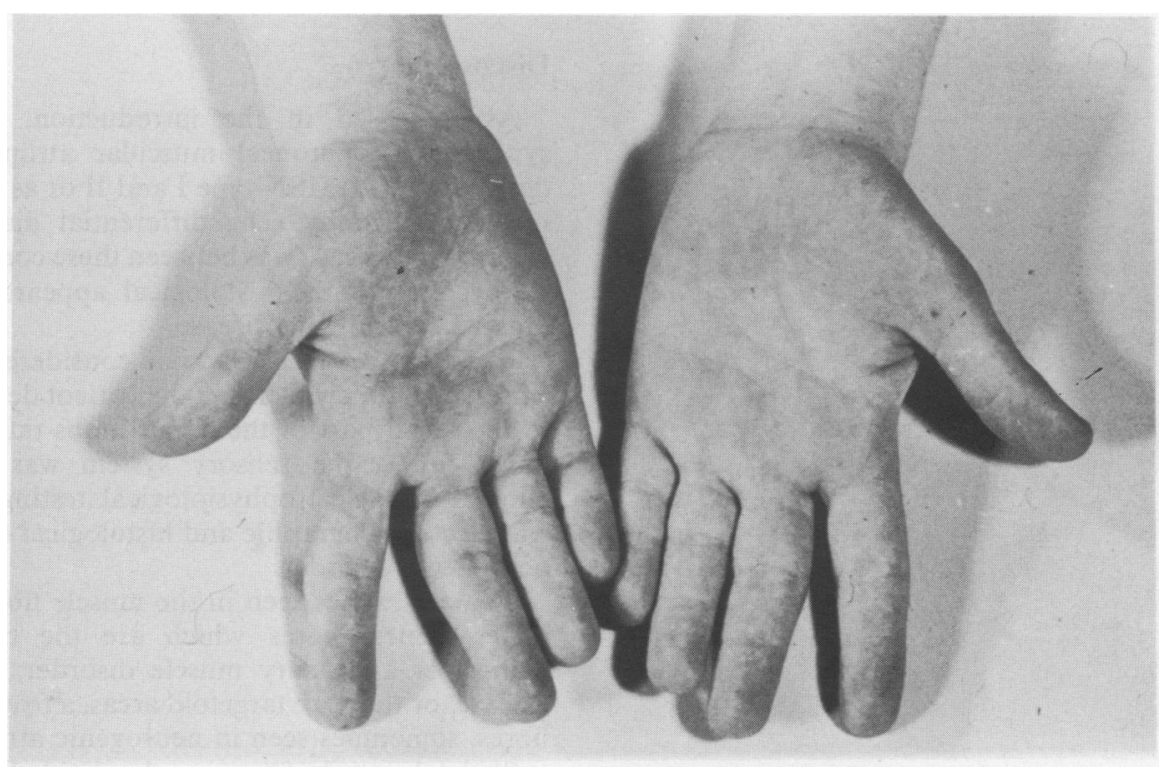

FIG. 2. Palmar aspect of the hands of the patient. There is wasting of thenar and hypothenare eminences. 


\section{Investigations}

The full blood count, urea and electrolytes, liver function tests, serum calcium and phosphorus were all normal. His creatine phosphokinase was $81 \mathrm{iu} . / 1$ (normal 0-50) and the 24-hr urine creatine was 1380 mmol (normal 0-400).

Electromyography showed no evidence of a myopathic process. However, sampling of several limb muscles showed continuous fibrillation with the muscles relaxed and fasciculation of either single or grouped units, some of large amplitude. These changes were most marked in the distal muscles. The motor conduction velocity of the median nerve was $40 \mathrm{~m} / \mathrm{s}$ (normal mean $55 \cdot 1 \mathrm{~m} / \mathrm{s}$, s.d. $\pm 5 \cdot 2$ ) and of the ulnar nerve was $31 \mathrm{~m} / \mathrm{s}$ (normal mean $59.4 \mathrm{~m} / \mathrm{s}$, s.d. $\pm 5 \cdot 0$ ). Testing for sensory function of the median and ulnar nerves at the wrist revealed no abnormalities. Motor conduction velocity for the lateral popliteal nerve (knee to ankle segment) was $37 \mathrm{~m} / \mathrm{s}$ (normal mean $52.0 \mathrm{~m} / \mathrm{s}$, s.d. \pm 4.0 ). The normal means and standard deviations are those quoted by Johnson (1980). A normal sural nerve action potential was recorded.

A biopsy of the left vastus lateralis muscle was examined by light and electronmicroscopy. There was prominent atrophy of groups of fibres-group fibre atrophy (Fig. 3). There were areas of mild endomyseal fibrosis and occasional intrafascicular fat cells. On transverse sections several fibres had pale central zones, best demonstrated on phosphorylase and on oxidative enzyme histochemical preparations

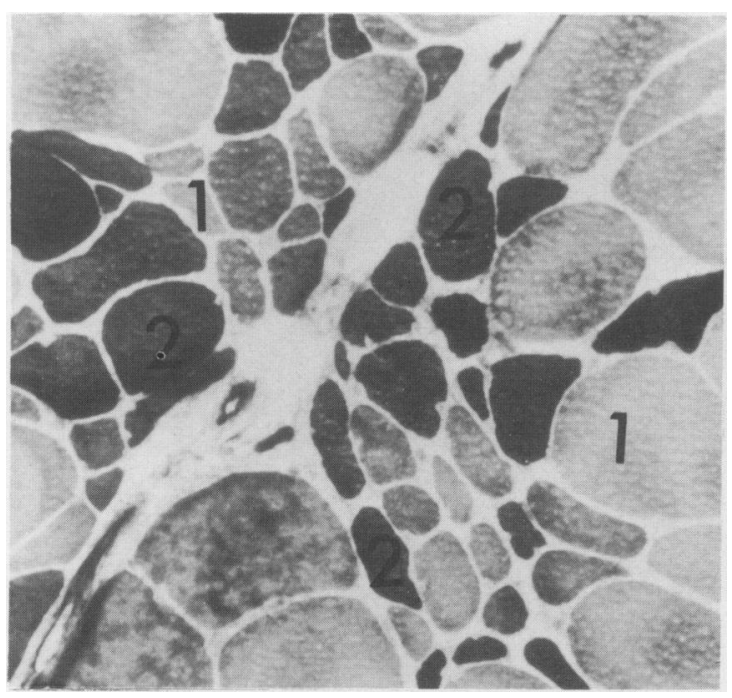

FiG. 3. Group fibre atrophy is seen and scattered atrophic fibres of both types 1 and 2 . ATPase at $9.4 \times 100$.

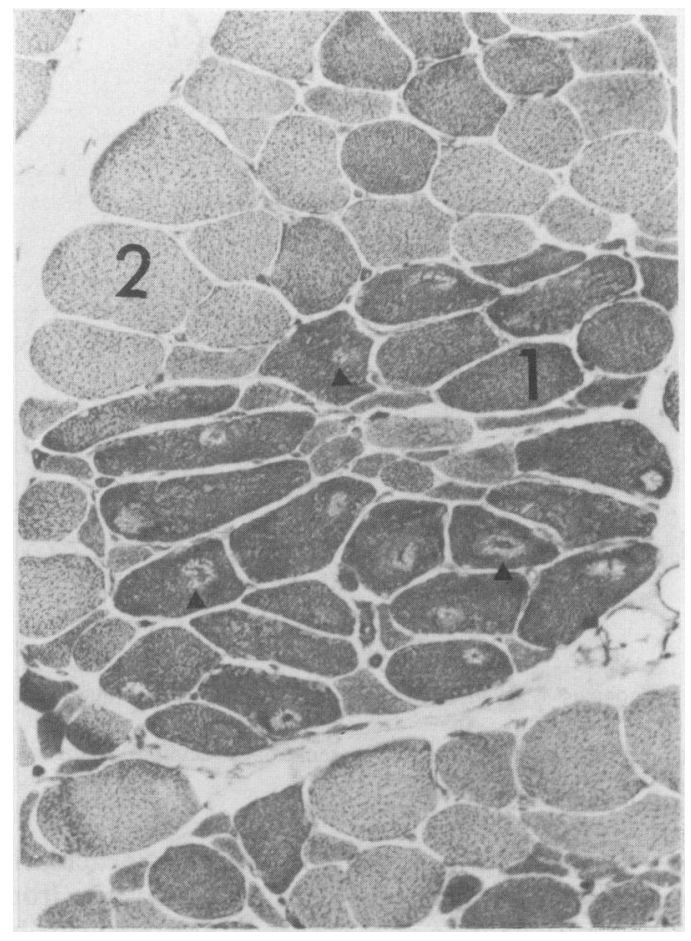

FIG. 4. In this field a large proportion of grouped type 1 fibres exhib 'core targetoid' zones (arrows). NADH-TR $\times 100$.

(Fig. 4). On electron microscopy these 'core zones' were sharply defined, devoid of organelles and had central electron dense material.

\section{Discussion}

As discussed in the introduction, the clinical syndrome of peroneal muscular atrophy may be designated as HMSN type I and II or as distal spinal muscular atrophy. The differential diagnosis considered in this case was between these conditions and, on account of the histological appearances of the muscle, central core disease.

A diagnosis of HMSN was considered untenable since the main problems in the patient described were in the distal part of the upper limbs rather than the lower limbs, the sensory system was normal on clinical and electrophysiological testing, and there was electromyographic and histological evidence of a neurogenic disorder.

The core zones seen in the muscle fibres could be either central cores which are the characteristic feature of a primary muscle disorder, central core disease, or they are targetoid areas, a feature of target fibres, sometimes seen in neurogenic atrophy.

Skeletal muscle fibres can be divided into types 1 and 2 , the former being rich in oxidative enzymes, the 
latter in glycolytic enzymes. Targetoid or core zones are seen only in type 1 fibres. In central core disease there is a preponderance of type 1 fibres and $90 \%$ of these exhibit central cores (Dubowitz and Brooke, 1973). These criteria were absent in this muscle biopsy so the diagnosis of central core disease was not tenable.

Target fibres in this number and distribution are a characteristic of denervating disease (Engel, 1961). Furthermore, the unequivocal and prominent group fibre atrophy is only seen in denervation atrophy. Thus the biopsy findings endorsed the neurophysiological criteria which pointed to a diagnosis of spinal muscular atrophy.

Clinically the case described fits the earlier description of distal spinal muscular atrophy recently reviewed by Harding and Thomas (1980). They noted onset of the disease during the first decade in 22 out of 34 cases, a higher incidence amongst males and variable patterns of inheritance. Sporadic cases were the commonest comprising $23 \%$ of the total. Given our patients's family history of pes cavus it is likely that there may be a genetically determined skeletal defect in this family. However, as both parents were normal on examination it cannot be a simple autosomal dominant defect.

The recent clinical classification of spinal muscular atrophy into 7 types by Pearn (1980) is most useful, but in some respects confusing. In particular, his reference to a distal form as 'Charcot-Marie-Tooth like' does not prove to be a homogeneous group when the cases quoted by him to support the separate existence of this subgroup are studied. For example, the cases quoted by Dyck and Lambert (1968) and by Pearn and Hudgson (1979) describe patients in whom the lower limbs bore the brunt of the disease. In contrast, the cases described by Meadows and Marsden (1969) and the first patient described by Magee (1960) showed the upper limbs to be predominantly involved. We suggest a more appropriate classification of this subgroup should be considered according to the clinical observations of Harding and Thomas (1980). They proposed that there are intermediate cases between the common form of distal spinal muscular atrophy which predominantly involves the legs and the much rarer variety with pure upper limb involvement, as described by Larder, Eadie and Tyner (1976) and O'Sullivan and McLeod (1978).

In the intermediate category there is marked weakness of the upper limbs with less severe problems in the lower limbs, as described in family 3 by Harding and Thomas (1980). Young and Harper (1980) described a young patient who is best classified in this category. He noted weakness in his hands followed shortly afterwards by lower limb weakness. He had normal sensory nerve conduction and slight slowing of motor conduction $(41 \mathrm{~m} / \mathrm{s})$ in the ulnar nerve. We suggest that it is in this unusual intermediate category of distal spinal muscular atrophy that the patient described in our paper may best be classified.

\section{Acknowledgments}

The authors are indebted to the late Dr Peter Croft for permission to report this case. We are grateful to Professor R. H. T. Edwards for the use of his laboratory facilities, to Dr Jennifer McDougall for electron microscopy and to Mrs Kathy Deed for typing and secretarial help.

\section{References}

Brust, J.C.M., LovelaCe, R.E. \& DeVI, S. (1978) Clinical and electro-diagnostic features of Charcot-Marie-Tooth syndrome. Acta Neurologica Scandinavia, 58, Suppl. 68, 1.

CHARCOT, J.M. \& MARIE, P. (1886) Sur une forme particulière d'atrophie musculaire progressive, souvent familiale, debutant par les pieds et les jambes, et atteignant plus tard les mains. Revue de Médecine (Paris), 6, 97.

DUBOWITZ, V. \& BROOKE, M.H. (1973) Muscle Biopsy: A Modern Approach, p. 254. W. B. Saunders, London.

DYCK, P.J. (1975) Inherited neuronal degeneration and atrophy affecting peripheral motor, sensory and autonomic neurons. In: Peripheral Neuropathy (Ed by Dyck, P.J., Thomas, P.K. \& Lambert, E.H.), Vol. 2, p. 285. W. B. Saunders, Philadelphia.

DYCK, P.J. \& LAMBERT, E.H. (1968) Lower motor and primary sensory neuron diseases with peroneal muscular atrophy. 1. Neurologic, genetic and electrophysiologic findings in hereditary polyneuropathies. Archives of Neurology (Chicago), 18, 603.

ENGEL, W.L. (1961) Muscle target fibres, a newly recognised sign of denervation. Nature (London), 191, 389.

HARDING, A.D. \& ThOMAS, P.K. (1980) Hereditary distal spinal muscular atrophy. A report on 34 cases and a review of the literature. Journal of the Neurological Sciences, 45, 337.

JoHnson, E.W. (1980) Practical Electromyography. Williams \& Wilkins, Baltimore.

LARDER, C.M., EADIE, M.J. \& TYNER, J.M. (1976) Hereditary motor peripheral neuropathy affecting the arms. Journal of the Neurological Sciences, 28, 389.

MAGEE, K.R. (1960) Familial progressive bulbospinal muscular atrophy. Neurology (Minneapolis), 10, 295.

MARTIN-SNEESENS, L. (1962) Formes à evolution trés prolonges de l'amyotrophie spinale de Werdnig-Hoffman. Journal de génétique humaine (Geneva), 11, 251.

MEADOWS, J.C. \& MARSDEN, C.D. (1969) A distal form of chronic spinal muscular atrophy. Neurology (Minneapolis), 19, 53.

O'SullivaN, D.G. \& MCLEOD, J.G. (1978) Distal chronic spinal muscular atrophy involving the hands. Journal of Neurology, Neurosurgery and Psychiatry, 41, 653.

PEARN, J.P. (1980) Classification of spinal muscular atrophies. Lancet, i, 919.

PeARn, J. \& Hudgson, P. (1979) Distal spinal muscular atrophy: A clinical and genetic study. Journal of the Neurological Sciences, 43 , 183.

Thomas, P.K. \& CALNE, D.B. (1974) Motor nerve conduction velocity in peroneal muscular atrophy: evidence for genetic heterogeneity. Journal of Neurology, Neurosurgery and Psychiatry, $37,68$.

Thomas, P.K., Calne, D.B. \& STewart, G. (1974) Hereditary motor and sensory polyneuropathy (peroneal muscular atrophy). Annals of Human Genetics, 38, 111.

TOOTH, H.H. (1886) The Peroneal Type of Progressive Muscular Atrophy. H.K. Lewis, London.

YOUNG, I.D. \& HARPER, P.S. (1980) Hereditary distal spinal muscular atrophy with vocal cord paralysis. Journal of Neurology, Neurosurgery and Psychiatry, 43, 413. 